\title{
Gelenkverschleiß erkennen, bevor der Schmerz beginnt
}

Mit einem Infrarot-Sensor wollen Forscher um den Ulmer Chemiker Prof. Dr. Boris Mizaikoff degenerative Veränderungen des Knorpels erkennen, bevor eine schmerzhafte Arthrose entsteht. Der Sensor soll im Zuge der minimal-invasiven Gelenkspiegelung eingesetzt werden. In etwas mehr als drei Jahren soll der „ArthroseScanner" marktreif sein.

Anlaufschmerzen, Morgensteifigkeit und eine verminderte Belastbarkeit: Arthrose schränkt die Lebensqualität der Patienten stark ein. Allerdings wird die Diagnose mittels bildgebender Verfahren oder Gelenkspiegelung oft erst gestellt, wenn der schützende Knorpel bereits stark abgetragen ist und womöglich schon die Gelenkflächen aufeinander reiben.

Dabei könnte ein Fortschreiten der Krankheit und letztlich der Ersatz des Gelenks in vielen Fällen verzögert werden, wenn Verletzungen des Knorpels frühzeitig erkannt und behandelt würden. Wissenschaftler um Prof. Dr. Boris Mizaikoff, Leiter des Ulmer Instituts für Analytische und Bioanalytische Chemie, entwickeln derzeit den Prototypen eines arthroskopischen Sensors im mittleren Infrarotbereich, der krankhafte Knorpelveränderungen bereits aufspürt, bevor eine schmerzhafte Arthrose entsteht.

Der neuartige Sensor detektiert molekulare Veränderungen im Zuge der Arthroskopie und könnte eine wesentliche Bereicherung für Patientenversorgung und Forschung sein: „Neben einer verbesserten unmittelbaren Diagnostik kann mithilfe dieser Messtechnik auch der Erfolg neuartiger Therapien überprüft werden", erklärt Mizaikoff. Zudem erhoffen sich die Wissenschaftler ein besseres Verständnis der Krankheitsentstehung und -entwicklung.

Die Ulmer Forscher bringen vor allem ihre Expertise im Bereich Infrarotspektroskopie und in der Miniaturisierung molekular-spezifischer Sensortechnologien ein. „Der neue
Sensor basiert auf einer Serie abstimmbarer Quantenkaskadenlasern, einem integrierten Strahlkombinator, Infrarot- Lichtwellenleiterfasern, sowie einem Sensorelement, das im mittleren Infrarotbereich zur Spektroskopie und Bildgebung dient. Eine Herausforderung ist die Integration all dieser Komponenten in ein hochkompaktes Format, das die tatsächliche Anwendung während des arthroskopischen Eingriffs erlaubt", so Mizaikoff. Ein miniaturisierter Prototyp hat im Vor- feld des Projekts bereits überzeugt: In Meniskusproben konnten krankhafte Veränderungen, aber auch atherosklerotische Ablagerungen an der Blutgefäßinnenseite erfolgreich detektiert und klassifiziert werden.

rheuma plus $2017 \cdot 16: 119$ https://doi.org/10.1007/s12688017-0141-3

๑) Springer-Verlag GmbH Austria 2017

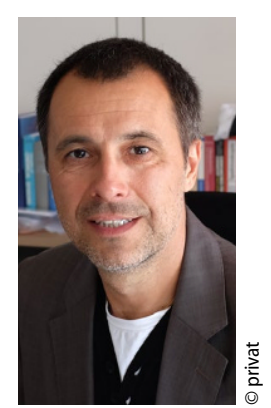

$\Delta$ Prof. Boris Mizaikoff

Quelle: idw
Hier steht eine Anzeige.

Springer 